\title{
MULTI-AGENT BASED CAPITAL MARKET MANAGEMENT SYSTEM: A DisTRIBUTED FRAMEWORK FOR TRADING AND REGULATION
}

\author{
Muhammed Kabir Ahmed ${ }^{1}$, Aliyuda Ali ${ }^{1}$ Ali Ahmad Aminu ${ }^{1}$ and Hassan Ibrahim ${ }^{2}$ \\ ${ }^{1}$ Department of Computer Science, Gombe State University, Gombe, Nigeria \\ ${ }^{2}$ Department of Computer Science, Gombe State Polytechnic, Gombe, Nigeria
}

\begin{abstract}
Stock Market plays a vital role in the economy of every nation. Having a transparent market may boost the confidence of not only stock brokers but also that of investors. One of the major problems that make investors to shy away from the market is lack of transparency. Another Problem which affect the market regulators is the lack of a system that enable them to check for compliance easily. In this work, an agent based distributed framework is presented. The idea behind the proposed system is that having one system that will serve all the market stake holders will guaranty strict compliance to the market rules, easier to manage and difficult manipulate by the market operators. The implementation of the proposed system followed Multi-Agent Software Engineering (MaSE) Methodology. The evaluation of the system show that, the distributed system developed using Java Agent Development Framework (JADE) is capable of addressing problems of reliability, compliance and transparency.
\end{abstract}

\section{KEYWORDS}

Stock Market, Multi-Agent, Distributed System.

\section{INTRODUCTION}

The capital market is a network of specialized financial institutions, series of mechanisms, processes and infrastructure that, in various ways, facilitate the bringing together of suppliers and users of medium to long-term capital for investment in socio-economic developmental projects. It embraces all the arrangements that facilitate the buying and selling of securities. The capital market has two segments, the Primary Market and the Secondary market. The primary market provides the avenue through which governments and corporate bodies raise fresh funds through the issuance of securities. It is otherwise known as the new issues market. Fresh funds can be raised through a combination of ways. These include public offers, rights issues and private placements. The secondary market provides investors the opportunity to buy or sell securities that were earlier issued in the primary market. The secondary market can be organized or unorganized. An organized market is a stock market with physical location, trading in designated (quoted) securities. Example of this is The Nigerian Stock Exchange. An unorganized market has no physical trading location but transactions are conducted mainly through telephone calls and the computer. It is called an Over-the-Counter-Market (OTC). The OTC trades mainly in unquoted securities. The distinguishing factor between the two segments is that in the primary market, the funds raised from investors go to the issuing entity, while in the secondary market; the proceeds from the transactions go to investors [1]. 
Nigerian stock market provides a major investment opportunity in country with the sole aim of improving the welfare of the citizen through wealth creation. The stock market has been identified as an institution that contributes to the economic growth of emerging economies, which also refers as a variable in explaining the economic growth in the most-developed ones. Stock exchanges play an increasingly important role, not only for channelling resources, but also for promoting reforms to modernize the financial sector legislation as is experienced in Nigeria and other emerging economies [1].

However despite the contributions of the stock market to the Nigerian economy, the market is still facing a lot of challenges which if not tackled, it will only affect the smooth running of the market but will also prevent the market from growing to reach its target stage so as to compete with other similar global markets. [2] Identify the challenges faced by individual investors in the course of their participation in the capital market which may be hindering the flow of funds to the market. A number of challenges ranging from inefficiencies of capital market operators, lack of information about quoted companies, the capital market crash, paucity of investible funds, low returns on investment, and lack of transparency in the market, among others were identified. Also the findings of [3] reveals that there is an underutilization of the Stock Exchange market due to poor enlightenment campaign, and lack of transparency and accountability on the part of the operators of the market. Another problem that has to do with the regulation of the market is the compliance issue in the stock broking firms. Most of the stock broking firms do not comply with the rules and regulation of the market. This is all as a result of deficiency of the regulators in the effective monitoring of the firms. E-Payment of dividend and bonuses to the beneficiaries by the firms at the appropriate time is also another reason that causes lack of confidence in the market by the shareholders.

It is believed that Multi-Agent Systems (MAS) can be applicable in a stock market environment due to the characteristics of the problems in the domain as it is very possible that the knowledge required to solve a problem is spatially distributed in different locations, the solution of a problem involves the coordination of the effort of different individuals with different skills and functions. MAS are very suitable in distributed and homogeneous environment.

An agent is a computer system within an environment and with an autonomous behavior made for achieving the objectives that were set during its design [4].

A multi-agents system is a system that contains a set of agents that interact with communications protocols and are able to act on their environment. Different agents have different spheres of influence, mainly because of their control (or at least an influence) on different parts of the environment. In some cases, these spheres of influence may overlap which causes dependency of reports between the agents [5].

Agent-based systems technology has generated a lot of excitement in recent years because of its promise as a new paradigm for conceptualizing, designing, and implementing software systems. This promise is particularly attractive for creating software that operates in distributed and open environments such as the internet. This Multi-Agent based System can be used in different application areas such as e-commerce and distributed information systems. In addition, MultiAgent based approach can be employed in a situation where accessing the most relevant information as easily, flexibly and timely as possible becomes necessary [6].

When designing systems that are complex, dynamic and stochastic in nature, simulation is generally recognized as one of the best design support technologies, and a valuable aid in the strategic and tactical decision making process. Due to the complexity of the real world, a simulation model can only be an approximation of the target system. The essence of the art of 
simulation modelling is abstraction and simplification. Only those characteristics that are important for the study and analysis of the target system should be included in the simulation model [7]. Multi-agent systems are promising as models of organizations because they are based on the idea that most work in human organizations is done based on intelligence, communication, co-operation, negotiation, and massive parallel processing [8].

Due to the contribution of Stock Market to the Nigerian economy, there is need to develop a distributed system that allow effective management of stocks by the market operators and at the same time providing shareholder and the market regulators with the avenue of monitoring the stocks to ensure the transparency of the market. There are a number of factors which point to the appropriateness of an Agent Based approach as agent is not a universal solution every problem that has to do with computing. So looking at the nature of stock market, the environment is open, or at least highly dynamic, uncertain and complex as well. In such environments, systems capable of flexible autonomous action are often suitable solution. Going by the attributes of an Agent such Autonomy (agents are able to decide their actions without the direct intervention of humans and others); Social Ability (agents interact with other agents in order to solve a problem by communication using some kind of agent-communication language); Reactivity (agents perceive their environments and respond to changes that occurs in them); Pro-activeness (agents has initiative, they do not act only in response to their environment); Multi-Agent Systems are capable of tackling most of the challenges faced in our stock market today.

\section{RELATED WORKS}

Recently agent technology is considered one of the most innovative technologies for the development of distributed software systems even though it has not being considered as a matured approach in the mainstream of software engineering. However a lot of work on agent technology has been done, many research results and applications have been presented, and some software products exists which have moved from the research community to the industrial community.

[9] Defines agent-based platform as a technical architecture that supports the development of agents and agent-based components, provides the environment in which agents can actively exist and operate to achieve their goals. [10] Suggested that the need of agent-based platforms comes from the fact that existing OOP development platforms and compilers do not support all facets of agent development such as they do not address the implementation of agent features, agent interaction rules, common knowledge base, and communication language. [11] Mentioned that traditional modeling tools are not applicable for complex system where there is need of analyzing and modeling of system in terms of their interdependencies. Agent-based platforms are the tools used for modeling and simulation of an agent-based system.

As agent orientation is a very broad field covering topics concerning inter alia agent organizations, agent behaviour as well as messaging it becomes obvious that most of these platforms focus on specific objectives and therefore cannot address all important aspects of agent technology equally well. Two important categories of platforms are middleware and reasoning oriented systems. The first category is mostly concerned with FIPA-related issues that address interoperability and various infrastructure topics such as white and yellow page services. Hence agent middleware is an important building block that forms a solid foundation for exploiting agent technology [12].

Due to the fact that many of the MAS characteristics are independent of the application, frameworks started to be utilized to facilitate the development of such systems. These frameworks provide the basic functionality of MAS, which allows the developers to concentrate 
International Journal of Managing Information Technology (IJMIT) Vol.13, No.2, May 2021

in the development of the agents. The main goal of the Foundation for Intelligent Physical Agents (FIPA) is to develop the standard implementation of the open, heterogeneous and interoperable agents. The foundation was created in 1996s and is also responsible to define some standard agents that many developers use to ensure the interoperability between MAS developed with generic frameworks [13]. Below some of the most widely use MAS platform are discussed.

Jade: is a software framework to make easy the development of multi-agent applications in compliance with the FIPA specifications. JADE can then be considered a middle-ware that implements an efficient agent platform and supports the development of multi agent systems. JADE agent platform tries to keep high the performance of a distributed agent system implemented with the Java language. In particular, its communication architecture tries to offer flexible and efficient messaging, transparently choosing the best transport available and leveraging state-of-the-art distributed object technology embedded within Java runtime [14].

[15] Presented a multi-agent based model of a simulated stock market within which active stock traders are modelled as heterogeneous adaptive artificial agents. The approach of integrating individual learning and social learning to co-evolve these artificial agents with the aim of evolving successful trading strategies was employed. The proposed model was tested on the British Petroleum (BP.L) share from the LSE (London Stock Exchange). On average, $80 \%$ of the artificial stock traders were able to trade using successful trading strategies which brings the investors higher returns compared to a baseline buy and-hold strategy.

Similarly in their further work, [15] developed a multi-agent based simulated stock market where artificial stock traders coevolved by means of individual and social learning and learn to trade stock profitably. The model was tested on a single stock (British Petroleum) from the LSE (London Stock Exchange) where artificial agents demonstrated dynamic learning behaviours and strong learning abilities. Different types of stocks from different sections of the stock market were also tested using the model. The results from the experiments show that the artificial traders demonstrate stable and satisfactory learning abilities during the simulation regardless of the different types of stocks.

[16] Introduced a new conceptual model representing the stock market dynamics. This model is essentially based on cognitive behaviour of the investors. In order to validate the model, an artificial stock market simulation based on agent-oriented methodologies was built. The proposed simulator is composed of market supervisor agent essentially responsible for executing transactions via an order book and various kinds of investor agents depending to their profile. The purposed of this simulation is to understand the influence of psychological character of an investor and its neighbourhood on its decision-making and their impact on the market in terms of price fluctuations. Interactions between investors and information exchange during a transaction reproduce the market dynamics and organize the multi-agent based pricing.

Also in another development, STOCK EXCHANGE MAS was implemented using the JADE platform. The system was run using actual values of the BM\&FBOVESPA index of 2004. In this simulation is established a communication between the agents that make up a STOCK BROKER, MARKET agent and other broker's COMMUNICATOR agents. At the start of the simulation the MARKET agent sends to all COMMUNICATOR agents (which are registered on the platform) a message informing the stock index value, characterizing the opening of trading. Thereafter every STOCK BROKER shall perform its flow to make decisions and make deals with the MARKET. During the execution of the simulation it is possible to see that agents gradually converge to similar behaviours. In early trading, when the index is down, some agents hold the majority shares and start selling the shares it owns. In the middle of the year with the onset of high index, the agents start to buy shares in small numbers, increasing the number of agents with this 
International Journal of Managing Information Technology (IJMIT) Vol.13, No.2, May 2021

behaviour as the index increases at each interaction. It is perceived that agents with low permittivity are less influenced by others. The agents with high permittivity are more susceptible to the decision of others, thereby mimicking the behaviour of majority of the agents assigning low relevance for the stock index [13].

According to [17] the regulatory organization in each country should be responsible for overseeing the health and stability of the overall financial system. The role of the systemic regulator should include gathering, analysing, and reporting information about significant interactions between and risks among financial institutions; designing and implementing systemically sensitive regulations, including capital requirements; and coordinating with the fiscal authorities and other government agencies in managing systemic crises.

On the aspect stock market regulation, even though most of the problems cited in this work have to do with the regulation but few or no work has been done in such research area. However, Multi-Agent Systems can play a significant role in the regulation as of the stock market especially in the area of monitoring and supervision.

Due to the distributive nature of the capital market, a computational intelligence technique called Ant Colony Optimization (ACO) which is suitable for solving distributed control problem was applied in [18] and [19] to get the most optimal solution. Both results provide a better prediction.

\subsection{Objectives of the Study}

The aim of this research is to model and simulate Nigerian Stock Market using Multi-Agent System. The specific objectives are to come up with a distributed system that can be used by the market stake holder which will enable the:

1) Market Regulators to monitor the market by ensuring to guarantee a fair and strict compliance to the rules and regulation by the firms as well as ensuring prompt payment of dividend to the respective shareholders.

2) Broker Agent trade using the optimal strategy by forecast the next day stock price using both technical and fundamental analysis.

3) Shareholder/Investor to monitor the progress of trading and also get the relevant information regarding market atmosphere.

\section{Methodology}

Multi-Agent Systems Engineering, a methodology for developing multi-agent system, will be used in the analysis and design of the proposed system. Ant Colony Optimization (ACO), a Meta heuristic technique was used to obtained optimal result of the prediction process. The development the decentralized Distributed System that will be used by all the market stake holders (Regulators, Stockbrokers and the shareholders) was done using Java Agent Development Framework (JADE).

\subsection{Analysis of MAS based Regulation and Prediction System}

The purpose of the MaSE Analysis phase is to produce a set of roles whose tasks describe what the system has to do to meet its overall requirements. 


\subsubsection{Capturing System Goals}

The first step in the Analysis phase is Capturing Goals which involve two sub-steps in Capturing Goals: identifying goals and structuring goals. First, we identified the goals from the initial system context (functional requirements). After that, the goals are analysed and structured in such a way that it can be used later in the Analysis phase.

From the goals derived from the above requirement, the architecture of the MAS to be developed is shown in Figure 1 where each of the agent perform its own task by collaborating with other agents under the coordination of the master agent. The Shareholder Agent is responsible for updating shareholder with important information regarding the stock as well as advising them on what to do based on the information at hand. Broker Agent also performs the same task with Shareholder agent to the Stock Brokers. Next is the Regulatory Agent which is responsible for ensuring strict compliance with the market rules by all the market operators. While Payment Agent is responsible for making payment of dividends to their respective beneficiaries and the Reporting Agent as it name implies is responsible for sending report on any bridge of rule by the operators. There is also a Decision Marker Agent (ACO) which performs the intelligent optimization regarding stock and make the market prediction.

The architecture also includes a Distributed Database which act as the central database and Global Knowledge based (GKB) for storing the professional knowledge on stock which is going to be accessed by all the agents.

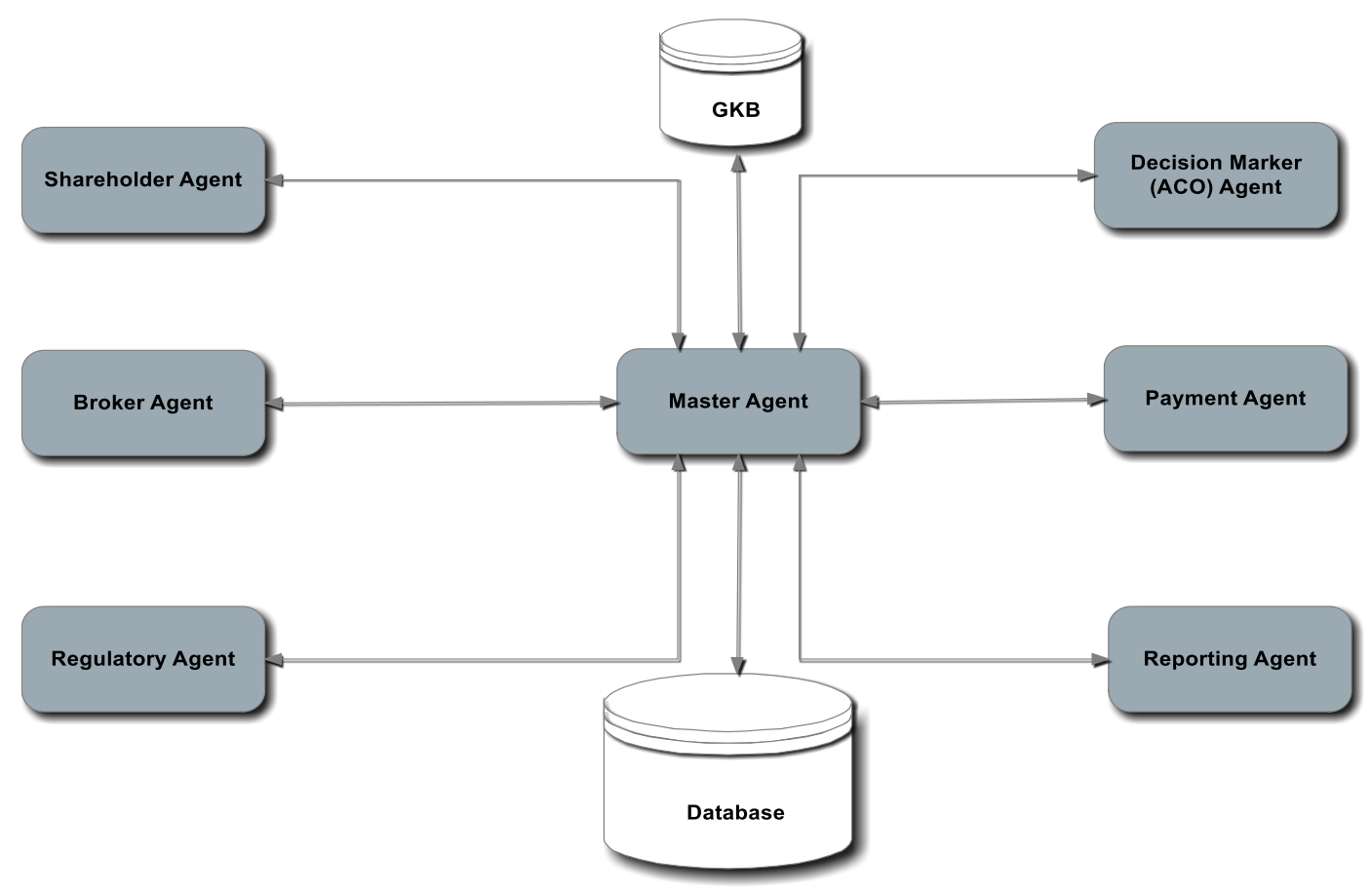

Figure 1: Multi-Agent Based Stock Market Regulation \& Prediction System

\subsubsection{Structuring System Goals}

The final step in Capturing Goals is structuring the goals into a Goal Hierarchy Diagram. While developing the goal hierarchy, the initial set of goals for their importance and inter-relationships has been studied. The Goal Hierarchy Diagram preserves such relationships, and divides goals 
International Journal of Managing Information Technology (IJMIT) Vol.13, No.2, May 2021

into levels of details and importance that are easier to manage and understand as shown in figure 2.

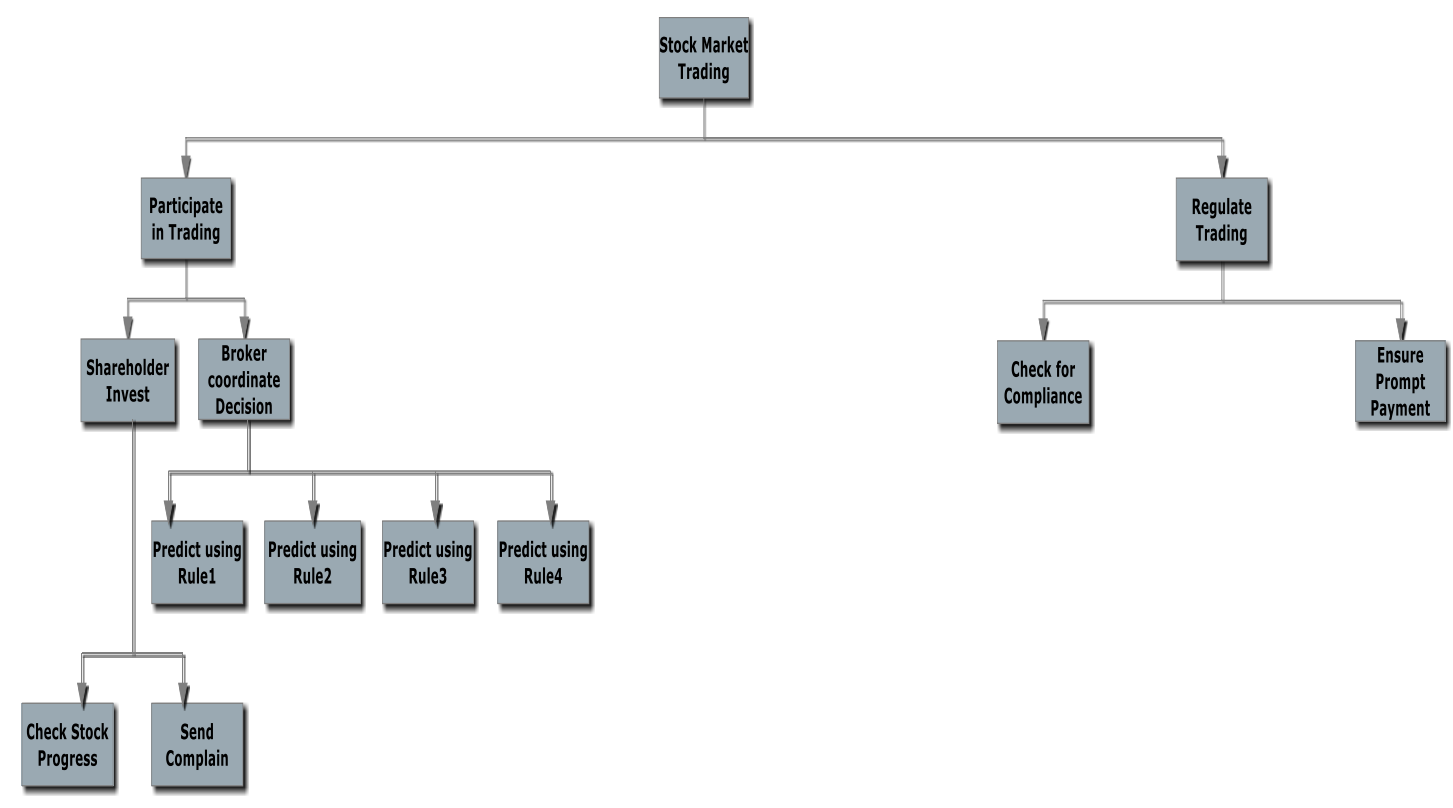

Figure 2: Goal Hierarchy Diagram

\subsubsection{Applying Use Cases}

The objective of the Applying Use Cases step is to capture a set of use cases from the initial system context so that it will be used to create a set of Sequence Diagrams. Actors in a stock market environment include Market Regulator, Broker, and Shareholders etc.

\subsubsection{Creating Sequence Diagrams}

A Sequence Diagram depicts the sequence of events that are transmitted between roles identified from use cases as shown in Figure 3. The boxes at the top of the diagram represent system roles and the arrows between the lines represent events passed between roles.

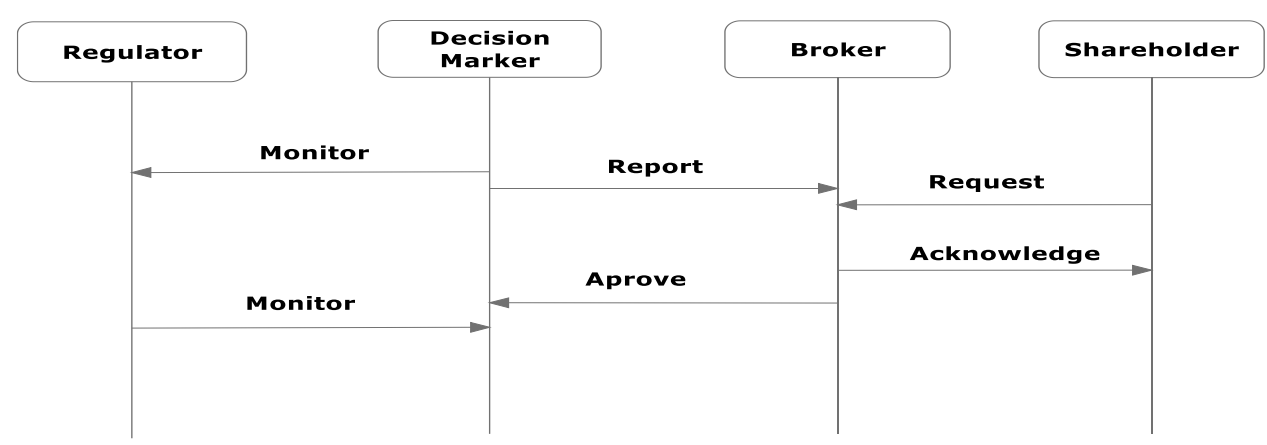

Figure 3: Sequence Diagram 


\subsubsection{Refining Roles}

The objective of the last step of the Analysis phase, Refining Roles, is to transform the structured goals and Sequence Diagrams into roles and their associated tasks, which are more suitable for designing multi-agent systems. Roles form the foundation for agent class definition and represent system goals during the Design phase. By using roles in this manner, the system goals are carried forward into the system design. The Role Identification Diagram of the proposed systems is shown in the Figure 4.

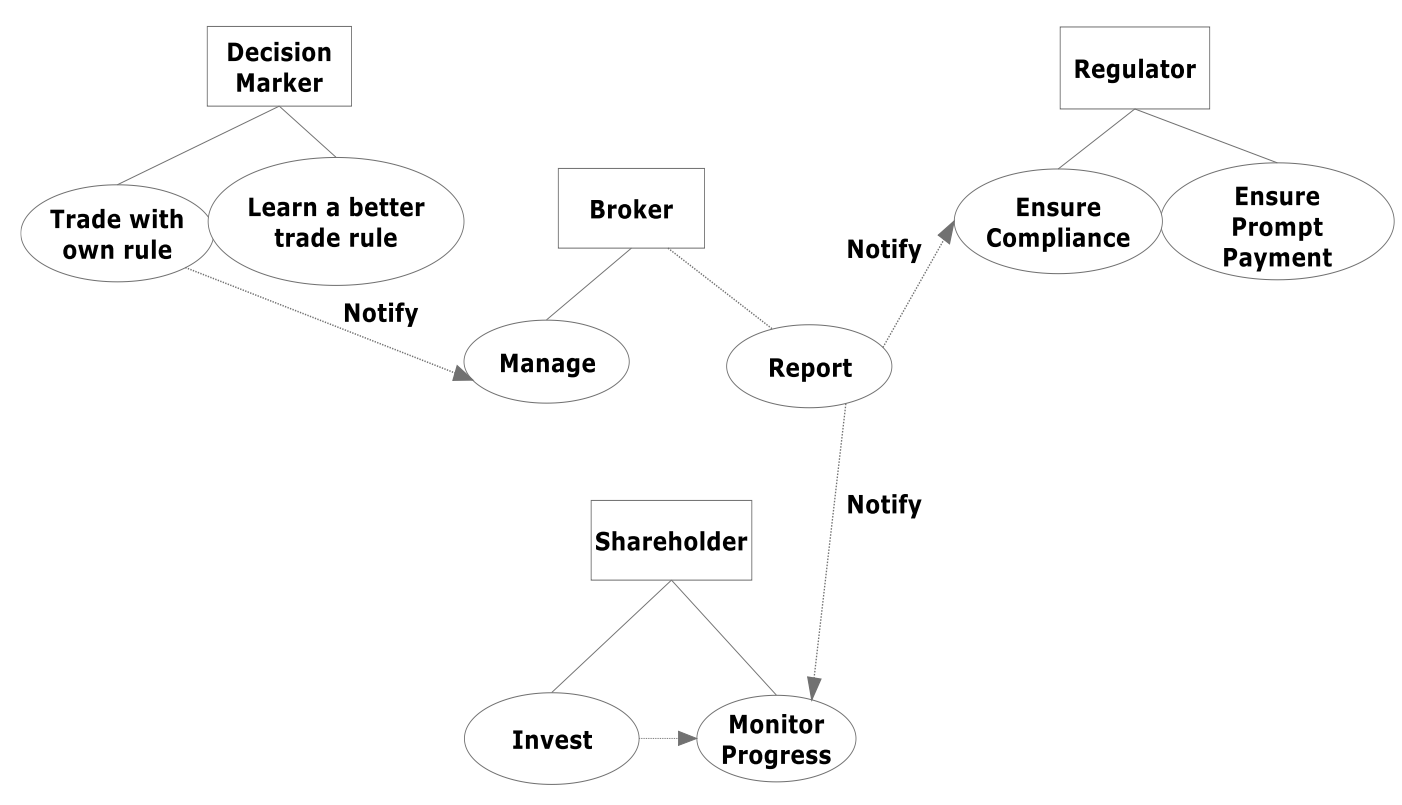

Figure 4: Role Identification Diagram

\subsubsection{Concurrent Task Diagram}

After roles are created, tasks are associated with each role that describes the behavior that the role must exhibit to successfully achieve its goals like the role of participating in trading using a particular strategy to achieve the goal of making profit. In general, a single role may have multiple concurrently executing tasks that define the required role behavior for example a broker may a detail of the market using a particular trading strategy and at the same time checking for the correct timing of either to buy or sell as shown in figure 5. Each task specifies a single thread of control that defines a particular behavior that the role may exhibit and integrates inter- as well as intra-role interactions. Concurrent tasks are specified graphically using a finite state automaton, which we refer to as a Concurrent Task Diagram. Similarly figure 6 shows the Concurrent Task Diagram for the Regulator Agent. 


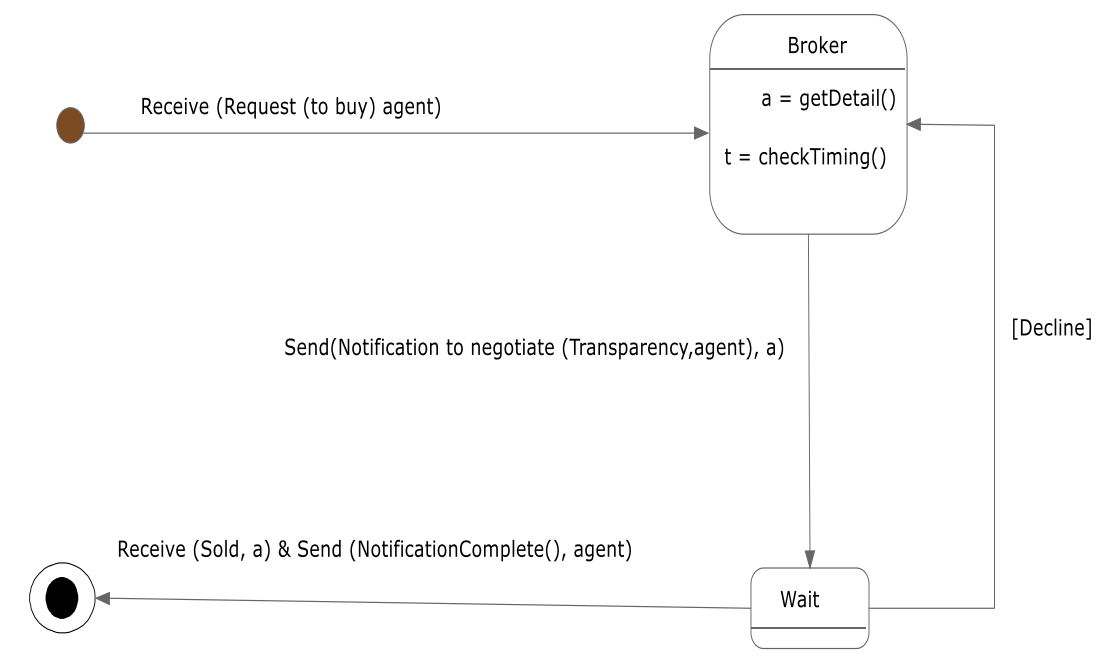

Figure 5: MABRPS Concurrent Task Diagram 1

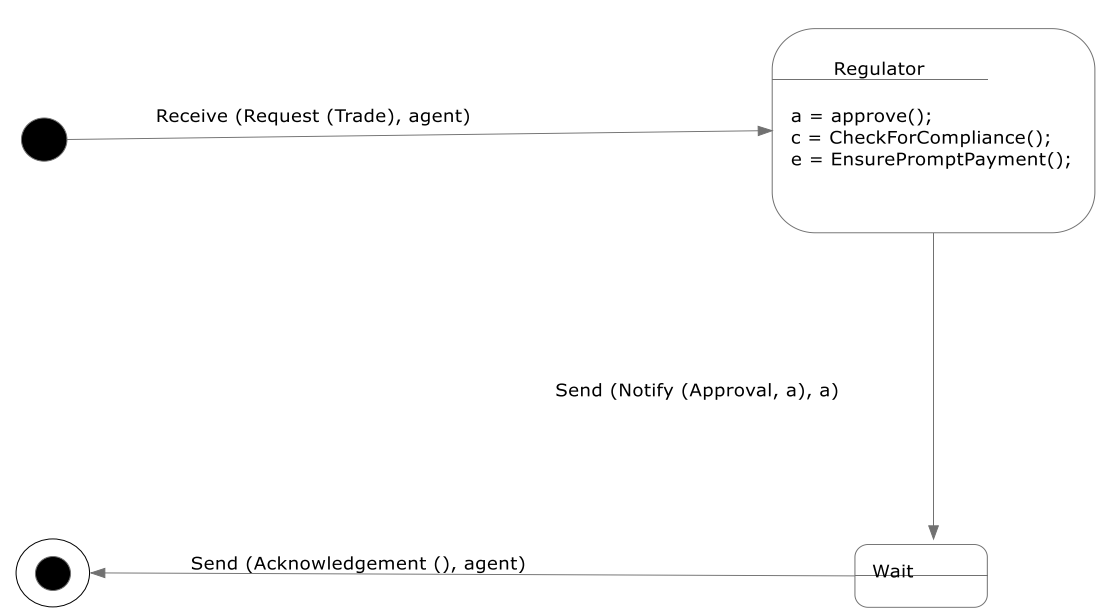

Figure 6: MABRPS Concurrent Task Diagram 2

\subsection{Design of Multi-Agent Based Regulation and Prediction System (MABRPS)}

The steps to follow in the designing of our proposed system with MaSE are Creating Agent Classes, in which we assign roles to specific agent types in the first step. In the second step, Constructing Conversations, the actual conversations between agent's classes are defined while in the third step, Assembling Agents Classes.

\subsubsection{Creating Agent Classes}

In the Creating Agent Classes step of the Design phase, agent classes are created from the roles defined in the Analysis phase. The end product of this phase is an Agent Class Diagram, which depicts the overall agent system organization consisting of agent classes and the conversations between them. An agent class is a template for a type of agent in the system and is analogous to an object class in object-orientation. An agent is an actual instance of an agent class. Figure 7 show the agent class diagram of the proposed system. 
International Journal of Managing Information Technology (IJMIT) Vol.13, No.2, May 2021

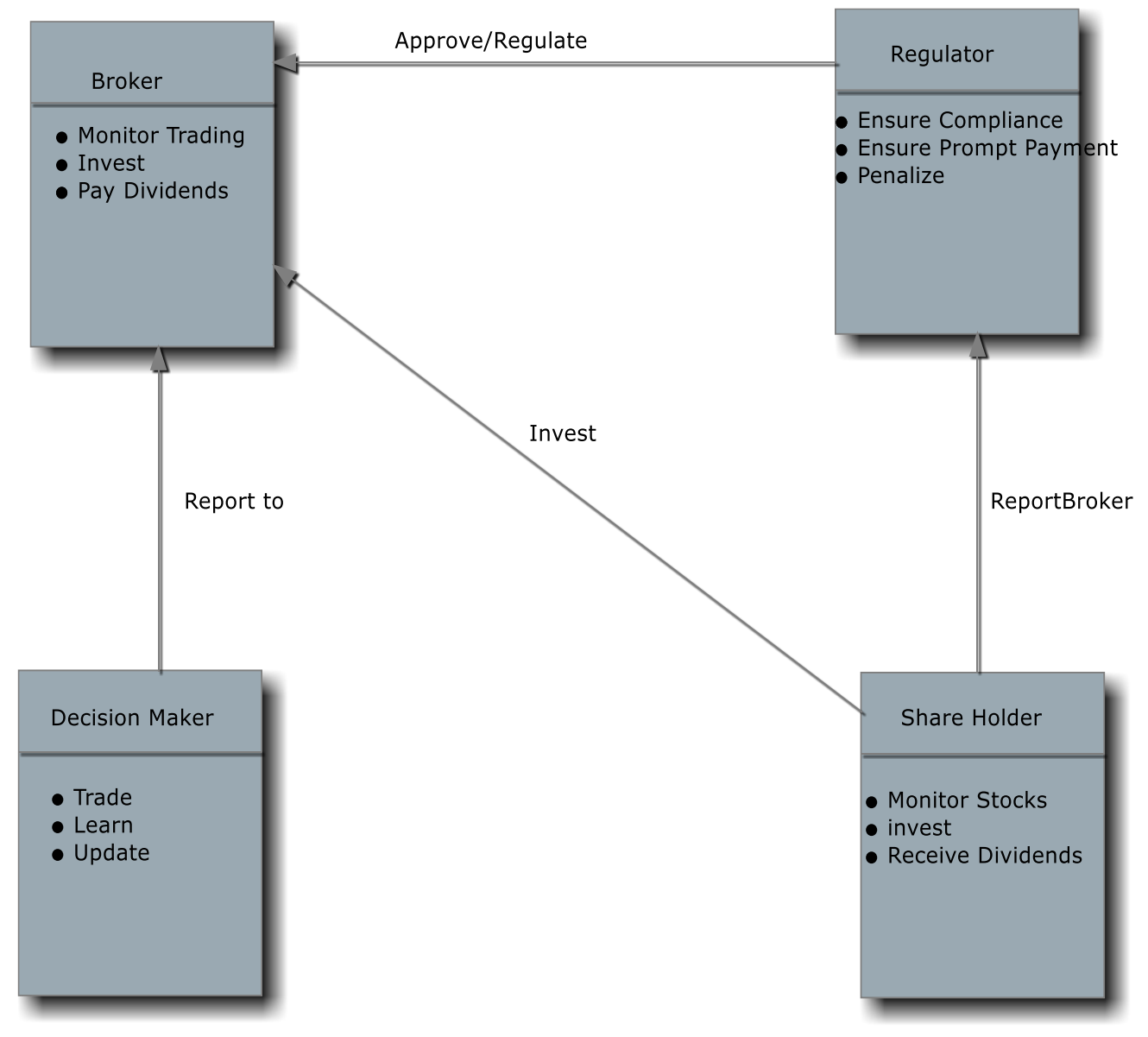

Figure 7: Agent Class Diagram of MABRPS

\subsubsection{Constructing Conversations}

Constructing Conversations is the next step in the MaSE Design phase. Up to this point, the communications has not been defined between agents beyond stating that they exist. The fact that a conversation must happen between two agents is known; the goal of this step is to actually define the details of these conversations. A Communication Class Diagram is a finite state automaton that defines the conversation states of the two participants the initiator and the responder which are shown in Figures 8 and 9. 
International Journal of Managing Information Technology (IJMIT) Vol.13, No.2, May 2021

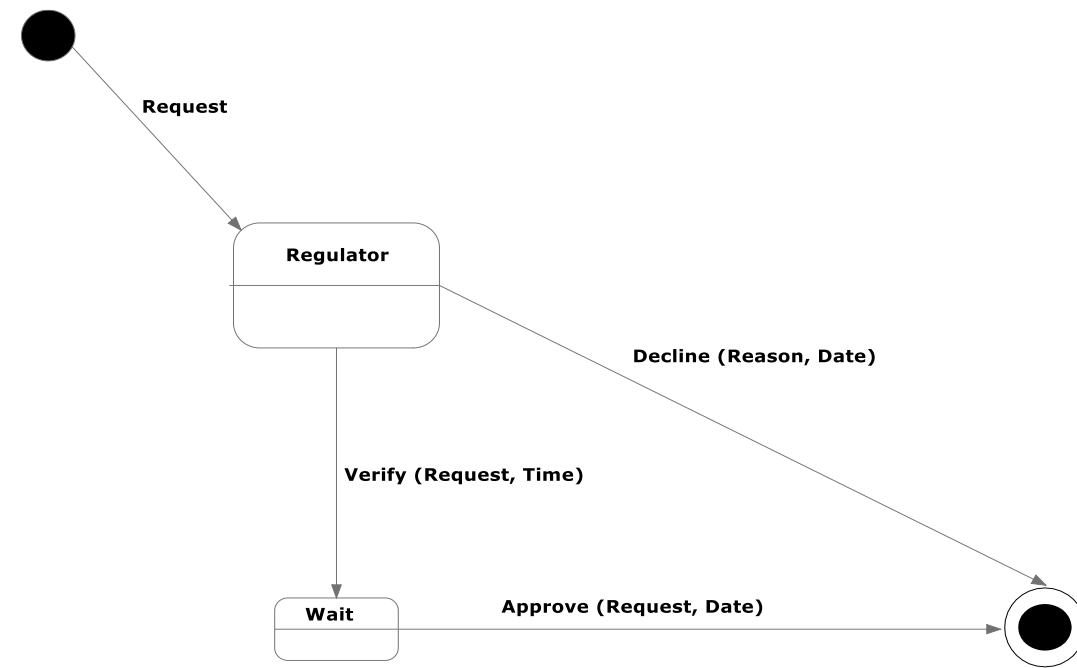

Figures 8: Communication between Broker and Regulator.

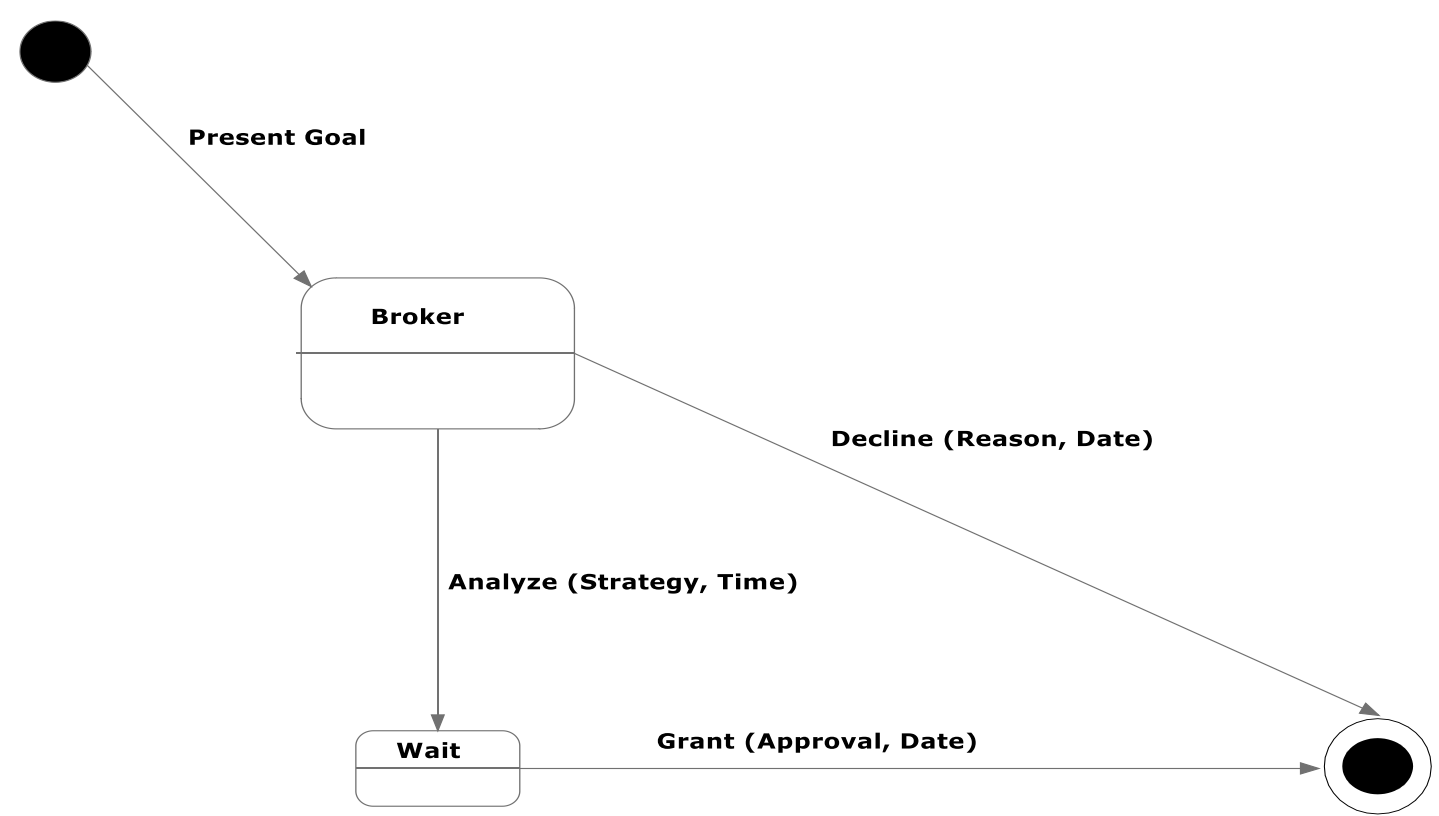

Figures 9: Communication between Decision Marker and Broker

\subsubsection{Assembling Agents}

During the Assembling Agents step of the Design phase, the internals of agent classes are created. This is accomplished via two sub-steps: defining the agent architecture and defining the components that make up the architecture. Designers have the choice of either designing their own architecture or using predefined architectures such as Belief-Desire-Intention (BDI) as shown in figure 10. 


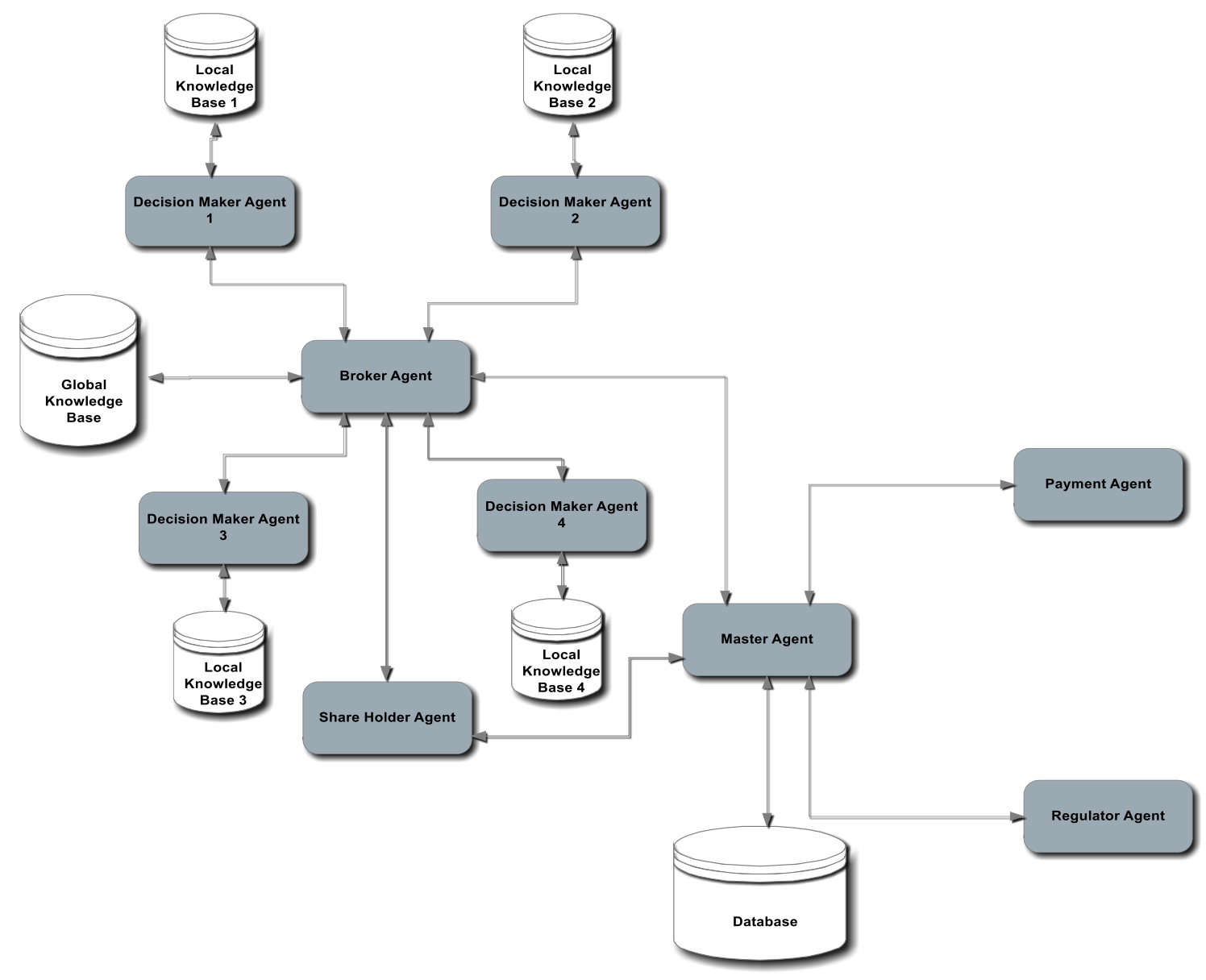

Figure 10: Macro Level System Architecture of MABRPS

\section{IMPLEMENTATION OF THE MULTi-AgENT BASED SySTEM}

Agent Based Simulation (ABS) a multi-agent based simulation technique, is going to be employ in this work. ABS model will describe the decision process of simulated actors at the micro level (agent's internal architecture). While Structures will emerge at the macro level as a result of the actions of the agent and their interactions with other agent.

Looking at the nature of stock market in general, it is also consist of heterogeneous, autonomous and proactive markets agents (Stock Brokers) that is why ABS is well suited to be used in the simulation of our proposed system. So our proposed multi-agent based system can be model based on intelligence, negotiation (when buying or selling stocks) and massive parallel processing (micro level optimization based on optimization of the rules using either technical or fundamental indicators. And learning as well (from agent with the best applicable strategy (best optimized rule).

Each agent behaviour is define by its own set of attribute values (either based on fundamental indicators, technical indicators or time series technique) which allow to model variation in the individual's behaviour and the simulation design is decentralized which allow agents to be proactive. 


\subsection{Decision Maker Agent 1 (Fundamental Ant Agent)}

The strategy of fundamental analysis is to compare the theoretical stock price with the market and then to see if the price is devalued or over-valued as shown in equation 4.1.

$$
r=\frac{p_{*_{i}}-p_{t-1}}{p_{t-1}}
$$

Where $P_{*_{i}}$ represents the theoretical stock price, $P t-1$ is the actual stock price on phase $\mathrm{t}-1$.

The value of $r$ may be positive or negative. $P_{*_{i}}$ is the average of the stock price during the phases.

When it is positive then the stock price is appreciate by that value while if it is negative then the stock is going to depreciate by the value also.

\subsection{Decision maker Agent 2 (Regression Agent)}

The Regression Agent uses Simple Regression Model (SRM) for the modelling. The original model in which the data was fitted is shown in equation 4.2 below:

$$
y_{i=} B_{0+} B_{1+} x_{i}
$$

$\mathrm{b}$ Where $\mathrm{y}_{\mathrm{i}}$ is the stock price for the ith day, $\mathrm{x}_{\mathrm{i}}$ is the ith day, $\mathrm{B}_{0}, \mathrm{~B}_{1}$, are the constants.

$$
\wedge y_{i}=B_{0+} B_{1+} x_{i}+e
$$

\subsection{Decision maker Agent 3 (Technical Analysis Agent)}

The technical analysis was done using five (5) days moving average in order to get the corresponding predicted stock prices for the various stocks under study.

The Predictions result obtained in section 4.1, 4.2 and 4.3 was used to get an optimal solution (prediction) using a computational intelligence technique called Ant Colony Optimization (ACO) which is suitable for solving distributed control problem as presented in our previous work [18] and [19]. The obtained optimal prediction is as follows: accuracy of 0.812500 , Sensitivity of 0.907407 and Specificity of 0.690476 . This optimal prediction strategy was used in the implementation of the system.

\section{Conclusions}

It can be seen that having one distributed system that will serve as the only platform for all the market stake holders can go a long way in checking for reliability and transparency of the market, also the framework can solve the issue of delay in the payment of dividend to beneficiaries (shareholders). On the issue of checking for compliance, the Security and Exchange Commission as the overall market regulator can easily check for compliance by the stock broking firms been the custodian of proposed system. The Investors as well as the shareholders can monitor their 
International Journal of Managing Information Technology (IJMIT) Vol.13, No.2, May 2021

stocks. In future work, the prototype system can be upgraded to cover other critical aspect of stock market such as risk analysis.

\section{REFERENCES}

[1] Abiola, A and Okodua, H. (2008). The Role of the Nigerian Stock Market In Achieving Vision 2020. Lagos Journal of Banking, Finance \& Economic Issues, Vol. 2 No. 1.

[2] Ikeobi, N. R. (2015). Challenges Faced by Individual Investors in the Nigerian Capital Market. European Journal of Business and Management. Vol.7, No.23.

[3] Chijioke, M. E. (2011). The Impact of Capital Market on The Nigerian Economy with Emphasis on the Role of the Nigerian Stock Exchange (Master's Thesis).

[4] Wooldridge, J. M. (1999). Intelligent agents, Multiagent Systems: a Modern Approach to Distributed Artificial Intelligence. Wiley \& Sons.

[5] Wooldridge, M. (2002). An Introduction to Multi-Agent Systems. Wiley \& Sons.

[6] Moreno, A. (2003). Medical Applications of Multi-Agent Systems. CiteSeerX.

[7] Siebers, P.O and Aickelin, U (2009) Introduction to Multi-Agent Simulation. School of Computer Science and IT (ASAP), University of Nottingham, Nottingham, UK.

[8] Gazendam, H.W.M. (1993). Theories about architectures and performance of multi-agent systems. In: III European Congress of Psychology. Tampere, Finnland.

[9] Bajracharya, K. and Duboz, R. (2013). Comparison Of Three Agent-Based Platforms On The Basis Of A Simple Epidemiological Model (Wip): SpringSim TMS-DEVS, ACM, 7.

[10] Serenko, A., and Detlor, B. (2002). Agent toolkits: A general overview of the market and an assessment of instructor satisfaction with utilizing toolkits in the classroom. (Masters thesis, Michael G. DeG- roote School of Business McMaster University).

[11] Macal, C. M., and North, M. J., (2006). Tutorial on agent- based modeling and simulation part 2: How to model with agents: InProceeding of the 2006 winter simulation conference (wsc) Monterey, CA: IEEE: 7383

[12] Braubach, L., Pokahr, A., and Lamersdorf , W. (2005). Jadex: A BDI-Agent System Combining Middleware and Reasoning: CiteSeer.

[13] Batista, A. M., Marietto, M. B., Botelho, W.T., Kobayashi, G., Alves, B. P., Castro, S. and T. Ruas, L. (2010). Principles of Agent-Oriented Programming: Centre of Mathematics, Computation and Cognition, Federal University of ABC (UFABC) Brazil, InTech.

[14] Bellifemine, F., Caire, G. and Greenwood D. (2007). Developing Multi-agent Systems with JADE: John Wiley \& Sons.

[15] Kendall, G. and Su, Y. (2006). A Multi-agent Based Simulated Stock Market - Testing on Different Types of Stock:School of Computer Science and IT ASAP Research Group University of Nottingham Nottingham, NG81BB gxk@cs.nott.ac.uk

[16] Kodia, Z.,.Ben Said, L, and Ghedira K.(2010) A Multi-Agent Based Pricing : A Virtual Stock Market Simulation. 8th International Conference of Modeling and Simulation - MOSIM'10Hammamet - Tunisia "Evaluation and optimization of innovative production systems of goods and services"

[17] Baily, M. N. and Mishkin, F. S. (2009). A Systemic Regulator for Financial Markets Squam Lake Working Group on Financial Regulation. Working P A P E Center for Geoeconomic Studies.

[18] Ahmed, M. K., Wajiga, G. M, Blamah, N. V and Modi, B. (2019). Stock Market Forecasting Using ant Colony Optimization Based Algorithm. American Journal of Mathematical and Computer Modelling 2019. 4(3).

[19] Ahmed, M. K., Wajiga, G. M and Blamah, N. V. (2019). Multi-Agent Based Stock Market Prediction Using Ant Colony Optimization Technique. Quest Journals Journal of Software Engineering and Simulation Volume 5 Issue 1 